\title{
Full-vectorial propagation model and modified effective mode area of four-wave mixing in straight waveguides
}

Guo, Kai; Friis, Søren Michael Mørk; Christensen, Jesper Bjerge; Christensen, Erik Nicolai; Shi, Xiaodong; Ding, Yunhong; Ou, Haiyan; Rottwitt, Karsten

Published in:

Optics Letters

Link to article, DOI:

10.1364/OL.42.003670

Publication date:

2017

Document Version

Peer reviewed version

Link back to DTU Orbit

Citation (APA):

Guo, K., Friis, S. M. M., Christensen, J. B., Christensen, E. N., Shi, X., Ding, Y., Ou, H., \& Rottwitt, K. (2017). Full-vectorial propagation model and modified effective mode area of four-wave mixing in straight waveguides. Optics Letters, 42(18), 3670-3673. https://doi.org/10.1364/OL.42.003670

\section{General rights}

Copyright and moral rights for the publications made accessible in the public portal are retained by the authors and/or other copyright owners and it is a condition of accessing publications that users recognise and abide by the legal requirements associated with these rights.

- Users may download and print one copy of any publication from the public portal for the purpose of private study or research.

- You may not further distribute the material or use it for any profit-making activity or commercial gain

- You may freely distribute the URL identifying the publication in the public portal 


\title{
Full-vectorial propagation model and modified effective mode area of four-wave mixing in straight waveguides
}

\author{
Kal Guo ${ }^{1,2, *}$, Søren M. M. Frils ${ }^{2}$, Jesper B. Christensen ${ }^{2}$, Erik N. Christensen ${ }^{2}$, Xiaodong \\ $\mathrm{ShI}^{2}$, Yunhong Ding ${ }^{2}$, Haiyan Ou ${ }^{2}$, and Karsten Rottwitt ${ }^{2}$ \\ ${ }^{1}$ College of Opto-electronic Science and Engineering, National University of Defense Technology, Deya Road 109, Changsha, Hunan, China \\ ${ }^{2}$ Department of Photonics Engineering, Technical University of Denmark, Ørsteds Plads 343, Kgs. Lyngby, Denmark \\ *Corresponding author: guokai07203@hotmail.com
}

Compiled August 24, 2017

We derive from Maxwell's equations full-vectorial nonlinear propagation equations of four-wave mixing valid in straight semiconductor-on-insulator waveguides. Special attention is given to the resulting effective mode area, which takes a convenient form known from studies in photonic crystal fibers but has not been introduced in the context of integrated waveguides. We show that the difference between our full-vectorial effective mode area and the scalar equivalent often referred to in the literature may lead to mistakes when evaluating the nonlinear refractive index and optimizing designs of new waveguides. We verify the results of our derivation by comparing to experimental measurements in a silicon-on-insulator waveguide taking tolerances on fabrication parameters into account. () 2017 Optical Society of America

OCIS codes: (130.3120) Integrated optics devices; (190.4380) Nonlinear optics, four-wave mixing.

http://dx.doi.org/10.1364/ao.XX.XXXXXX

Nonlinear optical effects in integrated waveguide structures make possible a number of key features for optical signal processing such as lasing, amplification [1], and frequency conversion [2]. Being important building blocks for photonic integrated circuits, these features have been demonstrated extensively in a wide range of materials that allow high field confinement and hence strong nonlinear interaction $[3,4]$. Four-wave mixing (FWM) facilitates many desirable functionalities for optical signal processing such as phase and amplitude modulation [5], supercontinuum generation [6], photon-pair generation [7], simultaneous amplification and frequency conversion [8], and noise-less frequency conversion based on the special configuration of Bragg scattering [9]. Hence, a solid theoretical understanding of these processes, which is easily compared to experimental results, is important.

Comprehensive models of third-order nonlinear processes in integrated, high index-contrast waveguides have been presented earlier, yet some ambiguity of the evaluation of the waveguide nonlinearity is evident in the literature. Examples include definitions of the effective mode area containing the longitudinal component of the Poyntings vector [10], which hides information about the waveguide in the relation between the electric and magnetic field; intensity-based definitions of the field amplitude that do not explicitly give an effective mode area [11], which complicates comparison to experiments; factorizations of the nonlinear coefficient that produce an effective mode area with a fundamentally different physical interpretation than the usual (degree of nonlinearity is inversely proportional to effective mode area) $[12,13]$. In some works [14-16], it is assumed that the effective mode area can be approximated by the scalar version known from weakly guiding optical fibers [17], but this approach may be inaccurate up to a factor of 2 [12]. The latter conclusion has been verified in a bismuth suspended core fiber [18] but to the best of our knowledge not in an integrated waveguide.

In this letter, we derive full-vectorial propagation equations for pump-degenerate FWM directly from Maxwell's equations, valid in straight semiconductor-on-insulator waveguides using the approach of [19]. These equations take the same simple form as those known from highly nonlinear optical fibers [17] and all dependencies on the waveguide parameters are limited to the phase mismatch and the full-vectorial effective mode area, thus following the usual convention of leaving the nonlinear refractive index as a material parameter. We verify the result of our derivation by comparing to a simple silicon-on-insulator FWM experiment taking the tolerances on fabrication parameters into account; only the free carrier lifetime is left as a fitting parameter. Our experiment confirms that the scalar effective mode area underestimates the nonlinearity of the waveguide.

To derive propagation equations of FWM, we expand the physical electric and magnetic fields in a set of continuous wave frequency components

$$
\left\{\begin{array}{c}
\mathbf{E}(\mathbf{r}, t) \\
\mathbf{H}(\mathbf{r}, t)
\end{array}\right\}=\frac{1}{2} \sum_{n} \frac{A_{n}(z)}{N_{n}}\left\{\begin{array}{c}
\mathbf{F}(x, y) \\
\mathbf{U}(x, y)
\end{array}\right\} \exp \left(-i \omega_{n} t+i \beta_{n} z\right)+\text { c.c., }
$$

where $n$ is the summation index, $\mathbf{r}=\{x, y, z\}$ is the position coordinate, and $A_{n}$ is the complex amplitude of both the electric and the magnetic fields that varies along the longitudinal direction $z . \mathbf{F}_{n}$ and $\mathbf{U}_{n}$ are the field distribution functions of the electric and the magnetic fields, respectively, in the transverse dimension $S$. $\beta_{n}$ is the propagation constant at frequency $\omega_{n}$, 
while c.c. is the complex conjugate. The normalization factor $N_{n}$, which is defined as

$$
N_{n}^{2}=\frac{1}{4} \int_{S}\left[\mathbf{F}_{n} \times \mathbf{U}_{n}^{*}+\mathbf{F}_{n}^{*} \times \mathbf{U}_{n}\right] \cdot \mathbf{z} d s,
$$

makes sure that $\left|A_{n}\right|^{2}$ equals the optical power $P_{n}$ in watts, where $\mathbf{z}$ is a unit vector pointing in the longitudinal direction in the waveguide. Following the same approach from Maxwell's equations as in [19], the propagation equation of the field amplitude at frequency $\omega_{n}$ becomes

$$
\partial_{z} A_{n}(z)=\frac{i \omega_{n}}{4 N_{n}} \int_{S} \mathbf{F}_{n}^{*} \cdot \mathbf{P}_{n}^{N L}(\mathbf{r}) d s,
$$

where $\partial_{z}$ denotes differentiation with respect to $z$ and $\mathbf{P}_{n}^{N L}(\mathbf{r})$ is the frequency expansion coefficient at frequency $\omega_{n}$ of the Kerr-induced nonlinear polarization

$$
\mathbf{P}^{N L}(\mathbf{r}, t)=\frac{1}{2} \sum_{n} \mathbf{P}_{n}^{N L}(\mathbf{r}) \exp \left(-i \omega_{n} t+i \beta_{n} z\right)+\text { c.c. }
$$

The definition of the normalization factor $N_{n}$ in Eq. (2) contains both the electric and the magnetic field distribution functions, which inconveniently hides information about the waveguide in the relation between $\mathbf{F}_{n}$ and $\mathbf{U}_{n}$. A better understanding of the nonlinear properties of the waveguide is gained by rewriting Eq. (2) to $[13,20]$

$$
N_{n}^{2}=\frac{c}{2 n_{g}} \int_{S} n_{w}^{2}(x, y)\left|\mathbf{F}_{n}\right|^{2} d s,
$$

where $n_{g}$ is the group index of the guided mode, and $n_{w}(x, y)$ is the transverse refractive index distribution of the waveguide. The latter cannot be extracted from the integration in strongguiding waveguides with large refractive index contrast.

In the simple configuration of degenerate FWM, two pump (p) photons at frequency $\omega_{\mathrm{p}}$ annihilate, creating a pair of signal (s) and idler (i) photons at frequencies $\omega_{\mathrm{s}}$ and $\omega_{\mathrm{i}}$, respectively, thus fulfilling the energy conservation, $2 \omega_{\mathrm{p}}=\omega_{\mathrm{s}}+\omega_{\mathrm{i}}$. The Kerrinduced nonlinear polarization may, assuming an instantaneous electronic response, be written as the tensor product

$$
\mathbf{P}^{N L}=\epsilon_{0} \chi^{(3)}: \text { EEE. }
$$

where $\chi^{(3)}$ is the third-order susceptibility tensor, which is thus assumed independent of frequency in the bandwidth under consideration. Typically, the four wave components are coupled into a waveguide in the same polarization state, and within the narrow spectral range of phase matching all four waves may be assumed to have the same electric field distribution function $\mathbf{F}=\mathbf{F}_{p}=\mathbf{F}_{s}=\mathbf{F}_{i}$, which implies that the normalization factor $N_{n}$ is equal for all $n \in \mathrm{p}, \mathrm{s}$, i. Hence, the nonlinear induced polarization at the pump frequency becomes

$$
\begin{aligned}
\mathbf{P}_{p}^{N L}=[ & \left.\left(\left|A_{\mathrm{p}}\right|^{2}+2\left|A_{\mathrm{s}}\right|^{2}+2\left|A_{\mathrm{i}}\right|^{2}\right) A_{\mathrm{p}}+2 A_{\mathrm{s}} A_{\mathrm{i}} A_{\mathrm{p}}^{*} \exp (i \Delta \beta z)\right] \\
& \times \frac{\epsilon_{0}}{4 N^{3}} \chi_{c}^{(3)}\left[2|\mathbf{F}|^{2} \mathbf{F}+(\mathbf{F} \cdot \mathbf{F}) \mathbf{F}^{*}\right],
\end{aligned}
$$

where $\Delta \beta=\beta_{s}+\beta_{i}-2 \beta_{p}$ is the linear phase mismatch and it is assumed that only $\chi_{c}^{(3)}=\chi_{\text {iiii }}^{(3)}$ for $i=x$ or $i=y$ is nonzero. By inserting Eq. (7) (and the corresponding expressions for the signal and the idler nonlinear induced polarizations) into Eq. (3), we get the following propagation equations

$\partial_{z} A_{\mathrm{p}}=i \gamma_{e}\left[\left(\left|A_{\mathrm{p}}\right|^{2}+2\left|A_{\mathrm{s}}\right|^{2}+2\left|A_{\mathrm{i}}\right|^{2}\right) A_{\mathrm{p}}+2 A_{\mathrm{s}} A_{\mathrm{i}} A_{\mathrm{p}}^{*} \exp (i \Delta \beta z)\right]$,

$$
\partial_{z} A_{j}=i \gamma_{e}\left[\left(\left|A_{j}\right|^{2}+2\left|A_{\mathrm{p}}\right|^{2}+2\left|A_{k}\right|^{2}\right) A_{j}+A_{\mathrm{p}}^{2} A_{k}^{*} \exp (-i \Delta \beta z)\right]
$$

for $j, k \in\{\mathrm{s}, \mathrm{i}\}$ and $k \neq j$, and the nonlinear coefficient is defined as

$$
\gamma_{e}=\frac{\omega \epsilon_{0} n_{g}^{2}}{4 c^{2}} \frac{\int_{S} \chi_{c}^{(3)} \mathbf{F}^{*} \cdot\left[2|\mathbf{F}|^{2} \mathbf{F}+(\mathbf{F} \cdot \mathbf{F}) \mathbf{F}^{*}\right] d s}{\left(\int_{S} n_{w}^{2}|\mathbf{F}|^{2} d s\right)^{2}},
$$

where $n_{g}$ is the group index at the frequency $\omega$ that represents all four wave components. Equations (8)-(10) describe singlemode degenerate FWM in a straight waveguide and have the same form as the scalar versions widely known from highly nonlinear optical fibers [17]. At this point, one may define an effective mode area and an average nonlinearity, as done in $[12,13]$, which has the property that the effective mode area is independent on the nonlinearity of the waveguide at the cost of the average nonlinearity being dependent on the waveguide geometry. While such a factorization is as general as Eq. (10) and requires no specification of the waveguide structure, it also breaks with the usual convention of the (average) nonlinear refractive index being a material parameter. Instead, we assume that $\chi_{c}^{(3)}$ is negligible in the cladding, which is usually a very good approximation. Under this assumption, the nonlinear coefficient can be written as

$$
\gamma_{e}=\frac{\omega n_{2}}{c A_{\mathrm{eff}}^{(f)}}+i \frac{\beta_{T}}{2 A_{\mathrm{eff}}^{(f)}},
$$

where the nonlinear refractive index and the two-photon absorption coefficient are identified as

$$
n_{2}=\frac{3 \operatorname{Re}\left(\chi_{c}^{(3)}\right)}{4 n_{c}^{2} \epsilon_{0} c}, \quad \beta_{T}=\frac{3 \omega \operatorname{Im}\left(\chi_{c}^{(3)}\right)}{2 n_{c}^{2} \epsilon_{0} c^{2}},
$$

respectively, where $n_{c}$ is the refractive index of the core; the full-vectorial effective mode area becomes

$$
A_{\text {eff }}^{(f)}=\frac{3}{n_{g}^{2} n_{c}^{2}} \frac{\left(\int_{S} n_{w}^{2}|\mathbf{F}|^{2} d s\right)^{2}}{\int_{c} \mathbf{F}^{*} \cdot\left[2|\mathbf{F}|^{2} \mathbf{F}+(\mathbf{F} \cdot \mathbf{F}) \mathbf{F}^{*}\right] d s},
$$

where the presence of $n_{g}$ explicitly shows the well-known slowlight enhancement of the waveguide nonlinearity. A similar expression for the effective mode area is known from studies in photonic crystal fibers [21], derived from a pertubative method, but it has to the best of our knowledge not before been derived directly from Maxwell's equations. Note that the usual convention of $n_{2}$ in Eq. (11) being a material parameter and the effective mode area of Eq. (13) being a waveguide parameter is kept; this implies that the effective mode area of Eq. (13) and the one introduced in [12], i.e.

$$
A_{\mathrm{eff}}^{(a)}=\frac{\left|\int_{S}(\mathbf{F} \times \mathbf{U}) \cdot \mathbf{z} d s\right|^{2}}{\int_{S}|(\mathbf{F} \times \mathbf{U}) \cdot \mathbf{z}|^{2} d s}
$$

are not equal, as we discuss below. If the waveguide has a small index contrast, i.e. $n_{g} \approx n_{w}(x, y) \approx n_{c}$, and the fields are linearly polarized, Eq. (13) reduces to the usual scalar effective mode area $A_{\text {eff }}^{(\mathrm{s})}=\left(\int_{S}|\mathbf{F}|^{2} d s\right)^{2} / \int_{S}|\mathbf{F}|^{4} d s$. In reaching the scalar effective 
mode area, it is assumed that the electric field is continuous over the core-cladding boundary of the waveguide, which is not valid on standard integrated platforms due to the high index contrast. Even so, the scalar effective mode area is used in the context of FWM in the literature [14-16].

To highlight the differences between $A_{\text {eff }}^{(f)}, A_{\text {eff }}^{(a)}$, and $A_{\text {eff }}^{(s)}$ and to understand their impact when optimizing a waveguide design, we plot all three in Fig. 1 versus the waveguide core width $W$; a silicon core of height $H=250 \mathrm{~nm}$ embedded in a silica cladding is used as example, and the effective mode areas are simulated at a wavelength of $1560 \mathrm{~nm}$ in the fundamental transverse electric $\mathrm{TE}_{01}$-mode; the black circles denote the minimum of each curve; the inset in Fig. 1 shows a cross section of the simulated waveguide.

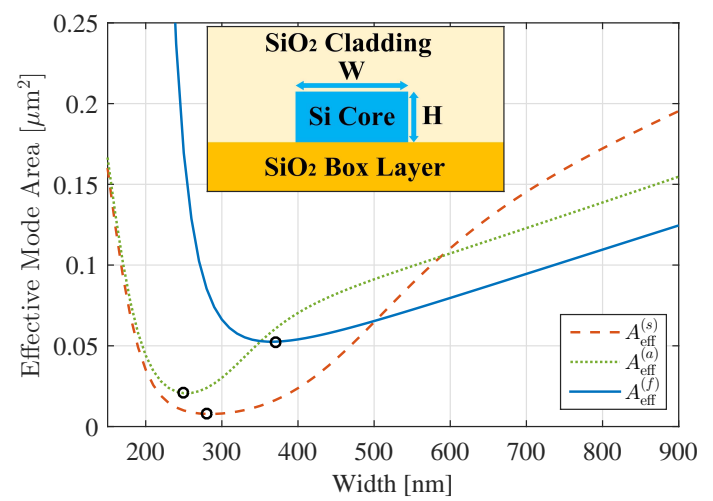

Fig. 1. Full-vectorial effective mode area $A_{\text {eff }}^{(\mathrm{f})}$ (blue solid), scalar effective mode area $A_{\text {eff }}^{(\mathrm{s})}$ (red dashed), and effective mode area of [12] $A_{\text {eff }}^{(\text {a) }}$ (green dotted) at $1560 \mathrm{~nm}$ versus waveguide width $W$. The inset is a cross section of the simulated waveguide.

Evidently $A_{\text {eff }}^{(\mathrm{f})}$ is larger than both $A_{\mathrm{eff}}^{(\mathrm{s})}$ and $A_{\mathrm{eff}}^{(\mathrm{a})}$ when $W$ is smaller than $500 \mathrm{~nm}$ and $350 \mathrm{~nm}$, respectively, and vice versa when $W$ is larger. The dramatically increasing $A_{\text {eff }}^{(\mathrm{f})}$ at small $W$ is due to the smaller integration area in the denominator of Eq. (13) thus reflecting the inverse relation to the nonlinearity of the waveguide. $A_{\text {eff }}^{(\mathrm{f})}$ is the smallest at large $W$ due to the dependence on the group index $n_{g}$ in Eq. (13), which also depends on $W$. The difference between the three curves highlights the importance of the definition of the effective mode area: Both $A_{\text {eff }}^{(\mathrm{f})}$ and $A_{\mathrm{eff}}^{(\mathrm{a})}$ are valid full-vectorial descriptions of the effective mode area of the field, though due to their different definitions they represent different physical properties of the waveguide; $A_{\mathrm{eff}}^{(\mathrm{s})}$ agrees only with $A_{\text {eff }}^{(\mathrm{a})}$ for unrealistically small dimensions but is otherwise wrong both in terms of value and where to locate the minimal effective mode area. Optimizing waveguide designs should thus either be based on minimizing $A_{\text {eff }}^{(\mathrm{f})}$ or maximizing the nonlinear coefficient associated with $A_{\text {eff }}^{(\text {a) }}$ in [12].

We verify the results of our derived model and confirm the incorrectness of the scalar mode area by a frequency conversion experiment in a silicon strip waveguide of dimensions $\mathrm{H} \times \mathrm{W}=$ $250 \mathrm{~nm} \times(450 \pm 10) \mathrm{nm}$. We compare the experimental results to numerical simulations of Eqs. (8)-(9) using $A_{\mathrm{eff}}^{(\mathrm{f})}$ and $A_{\mathrm{eff}}^{(\mathrm{s})}$, respectively, calculated to be $0.058 \mu \mathrm{m}^{2}$ and $0.042 \mu \mathrm{m}^{2}$.
To account for loss mechanisms in the silicon waveguide, it is necessary to include two more terms in Eq. (8) and Eq. (9), which are the linear loss $\alpha_{l}$, measured to be $2.0 \pm 0.3 \mathrm{~dB} / \mathrm{cm}$ through a cut-back procedure, and the frequency-dependent free carrier absorption (FCA) $\alpha_{f, j}$ given by

$$
\alpha_{f, j}=1.45 \cdot 10^{-21} \cdot\left(\frac{\lambda_{j}}{\lambda_{\text {ref }}}\right)^{2} \cdot \frac{\zeta_{e} \beta_{T}\left|A_{p}\right|^{4} \tau}{2 \hbar \omega_{p} A_{\text {eff }}^{(f)^{2}}}
$$

at wavelength $\lambda_{j}$ where $\lambda_{\text {ref }}=1550 \mathrm{~nm}$ is the reference wavelength, $\zeta_{e}$ is the polarization factor related to the intrinsic symmetry of silicon (1.25 for TE mode), and $\tau$ is the free carrier lifetime $[22,23]$. We use $n_{2}=6 \times 10^{-18} \mathrm{~m}^{2} / \mathrm{W}$ and $\beta_{T}=4.5 \times 10^{-12} \mathrm{~m} / \mathrm{W}[11,24]$ and keep $\tau$ as the only fitting parameter.

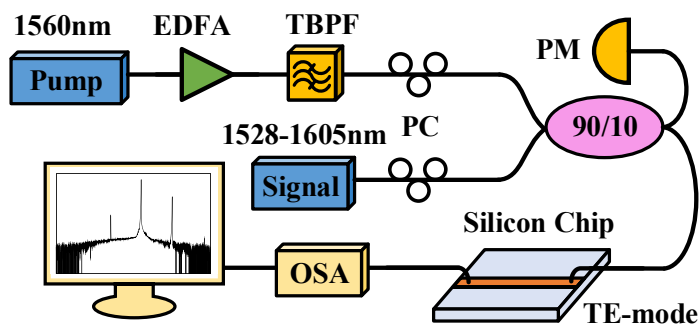

Fig. 2. Experimental set-up. EDFA: Erbium-doped fiber amplifier, TBPF: Tunable band-pass filter, PC: Polarization controller, OSA: Optical spectrum analyzer, PM: Power-meter.

A schematic of the experimental set-up is shown in Fig. 2. A continuous-wave (CW) pump laser at $1560 \mathrm{~nm}$ was amplified by an erbium-doped fiber amplifier (EDFA), and the amplified spontaneous emission (ASE) was suppressed by a tunable band-pass filter (TBPF). A CW tunable laser was used as signal. Polarization controllers (PCs) were employed to optimize the polarization, and thereby ensuring that the beam coupled into the waveguide through the photonic-crystal based grating coupler (PCGC) is in the $\mathrm{TE}_{01}$ mode and has a minimal coupling loss (4.5 dB per PCGC in our case) [25]. Through a 90/10 coupler, pump and signal were combined into a single mode fiber that was $75^{\circ}$ vertically aligned with the PCGCs, and a power-meter (PM) was used to monitor the input power. We used an optical spectrum analyzer (OSA) to measure the output conversion efficiency $\eta_{e, j}=P_{i}(L) / P_{\mathcal{S}}(L)$ versus signal wavelength $\lambda_{j}$ from $1528 \mathrm{~nm}$ to $1605 \mathrm{~nm}$.

Firstly, we measured $\eta_{e, j}$ in a $1.5 \mathrm{~cm}$-sample as seen in Fig. 3. By using a pump power of $18 \pm 0.5 \mathrm{dBm}$, we obtain a maximum conversion efficiency of $-15 \mathrm{~dB}$ with a $3 \mathrm{~dB}$ bandwidth of $28 \mathrm{~nm}$. This is fitted with our full-vectorial effective mode area $A_{\text {eff }}^{(\mathrm{f})}$ and the scalar effective mode area $A_{\text {eff }}^{(\mathrm{s})}$ in turn by varying $\tau$ from $1 \mathrm{~ns}$ to $30 \mathrm{~ns}$, and minimizing the root-mean-square error (RMSE) given by

$$
\text { RMSE }=\sqrt{\frac{1}{N} \sum_{j=1}^{N}\left(\eta_{n, j}-\eta_{e, j}\right)^{2}},
$$

where $\eta_{n, j}$ is the numerical prediction at wavelength $\lambda_{j}$. As shown in the inset in Fig. 3, the minimum RMSE is 0.86, using the full-vectorial model, resulting in a free carrier lifetime of $\tau=10 \mathrm{~ns}$ and with the scalar model the minimum RMSE is 1.53 for $\tau=16$ ns. In Fig. 3, the scalar model predicts values lower than the measured data near the pump wavelength, but higher 


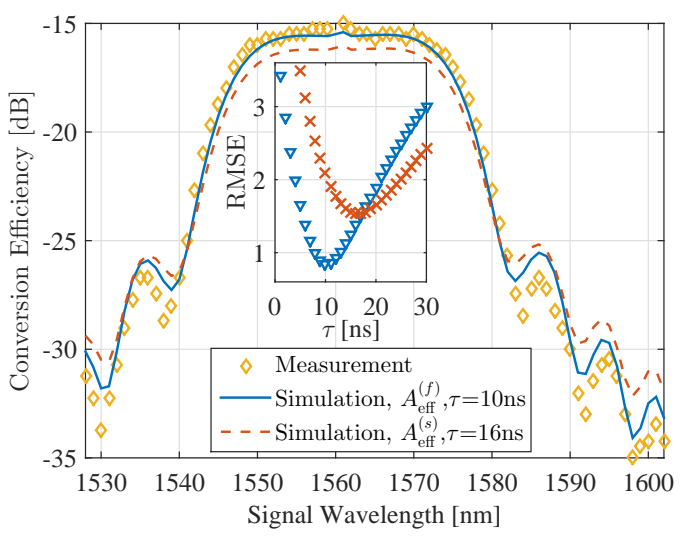

Fig. 3. Conversion efficiency versus signal wavelength, the measurements (yellow diamond), and the numerical predictions using $A_{\mathrm{eff}}^{(\mathrm{f})}$ (blue solid) and $A_{\mathrm{eff}}^{(\mathrm{s})}$ (red dashed) with $\tau$ of $10 \mathrm{~ns}$ and $16 \mathrm{~ns}$, respectively. The inset is the root-mean-square error between numerical and experimental data by fitting $\tau$ for $A_{\text {eff }}^{(\mathrm{f})}$ (blue triangles) and $A_{\text {eff }}^{(\mathrm{s})}$ (red crosses).

at the side lobes. As indicated by the RMSE value, a better fit is obtained with the full-vectorial model. A larger difference between the models could occur for different waveguide geometries as is evident from Fig. 1, since our waveguide of $450 \mathrm{~nm}$ width is close to the crossover point of the effective mode areas. To account for uncertainties in coupled pump power, propagation loss, and waveguide width, the models are fitted for all combinations within the uncertainty of power, loss, and width. For all the combinations the full-vectorial model exhibits a lower RMSE value than the scalar model.

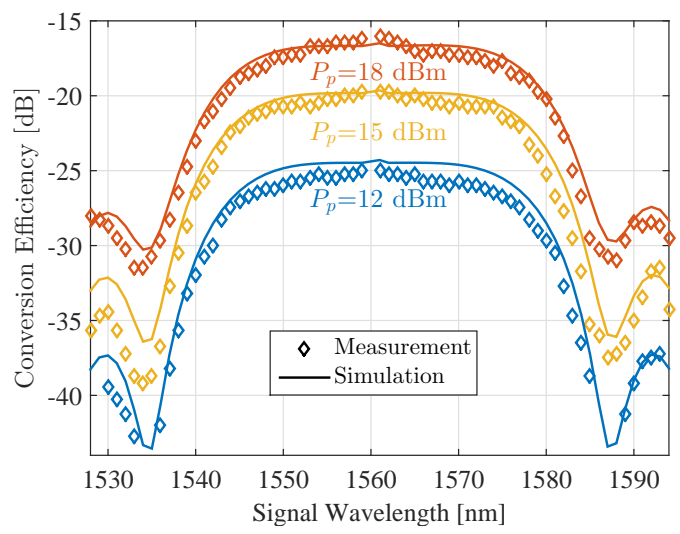

Fig. 4. Comparison of experimental (diamonds) and numerical (solid lines) conversion efficiency using $A_{\text {eff }}^{(\mathrm{f})}$ for $\tau=10 \mathrm{~ns}$ in a $1 \mathrm{~cm}$ waveguide; the pump powers are $P_{p}=18 \mathrm{dBm}$ (red), $P_{p}=15 \mathrm{dBm}$ (yellow) and $P_{p}=12 \mathrm{dBm}$ (blue).

To verify the comprehensiveness of the model with the acquired fit, $\tau=10 \mathrm{~ns}$, we repeat the experiment in a $1 \mathrm{~cm}$ sample on the same chip and with different pump powers. Figure 4 shows $\eta_{e, j}$ for $P_{p}=12 \mathrm{dBm}, 15 \mathrm{dBm}$, and $18 \mathrm{dBm}$. As expected, $\eta_{e, j}$ is smaller in the shorter waveguide and the bandwidth is broader. The maximal $\eta_{e, j}$ decreases for decreasing pump power. For all three values of the $P_{p}$ the derived model with the fullvectorial effective mode area and the fitted free-carrier lifetime $\tau=10 \mathrm{~ns}$ agrees very well with the experiment.
In conclusion, we derived full-vectorial nonlinear propagation equations of four-wave mixing valid in straight semiconductor-on-insulator waveguides. We introduced a convenient form of the effective mode area that follows the usual physical interpretation and at the same time captures the fullvectorial nature of light; it depends on the group refractive index, thus explicitly showing the well-known slow-light enhancement of the waveguide nonlinearity. We validated our derivation by comparing to a silicon-on-insulator frequency conversion experiment with only the free-carrier lifetime as a fitting parameter and found good agreement.

This work is supported jointly by the Chinese Scholarship Council, the Danish National Research Foundation Center of Excellence (DNRF123): Silicon Photonics for Optical Communications (SPOC), and The Danish Council for Independent Research, research grant: DFF-4184-00433.

\section{REFERENCES}

1. H. Rong, R. Jones, A. Liu, O. Cohen, D. Hak, A. Fang, and M. Paniccia, Nature 433, 725 (2005).

2. J. Leuthold, C. Koos, and W. Freude, Nat. Photon. 4, 535 (2010).

3. M. A. Foster, A. C. Turner, M. Lipson, and A. L. Gaeta, Opt. Express 16, 1300 (2008).

4. G. A. Siviloglou, S. Suntsov, R. El-Ganainy, R. Iwanow, G. I. Stegeman, D. N. Christodoulides, R. Morandotti, D. Modotto, A. Locatelli, C. De Angelis, F. Pozzi, C. R. Stanley, and M. Sorel, Opt. Express 14, 9377 (2006).

5. R. Salem, M. A. Foster, A. C. Turner, D. F. Geraghty, M. Lipson, and A. L. Gaeta, Nat. Photon. 2, 35 (2008).

6. I.-W. Hsieh, X. Chen, X. Liu, J. I. Dadap, N. C. Panoiu, C.-Y. Chou, F. Xia, W. M. Green, Y. A. Vlasov, and R. M. Osgood, Opt. Express 15, 15242 (2007).

7. K. Guo, E. N. Christensen, J. B. Christensen, J. G. Koefoed, D. Bacco, Y. Ding, H. Ou, and K. Rottwitt, Applied Physics Express 10, 062801 (2017).

8. M. A. Foster, A. C. Turner, J. E. Sharping, B. S. Schmidt, M. Lipson, and A. L. Gaeta, Nature 441, 960 (2006).

9. I. Agha, M. Davanço, B. Thurston, and K. Srinivasan, Opt. lett. 37, 2997 (2012).

10. C. Koos, L. Jacome, C. Poulton, J. Leuthold, and W. Freude, Opt. Express 15, 5976 (2007).

11. Q. Lin, O. J. Painter, and G. P. Agrawal, Opt. Express 15, 16604 (2007).

12. S. Afshar and T. Monro, Opt. Express 17, 2298 (2009).

13. S. Afshar, T. Monro, and C. M. de Sterke, Opt. Express 21, 18558 (2013).

14. T. J. Duffin, M. P. Nielsen, F. Diaz, S. Palomba, S. A. Maier, and R. F. Oulton, Optics letters 41, 155 (2016).

15. K. Gehlot and A. Sharma, Opt. Express 24, 4722 (2016).

16. L. An, H. Liu, Q. Sun, N. Huang, and Z. Wang, Appl. Opt. 53, 4886 (2014).

17. G. P. Agrawal, Nonlinear Fiber Optics (Elsevier, 2006), 4th ed.

18. S. Afshar, W. Q. Zhang, H. Ebendorff-Heidepriem, and T. Monro, Opt. Lett. 34, 3577 (2009).

19. M. Kolesik and J. Moloney, Phys. Rev. E 70, 036604 (2004).

20. A. W. Snyder and J. Love, Optical waveguide theory (Springer, 2012).

21. J. Lægsgaard, N. A. Mortensen, and A. Bjarklev, J. Opt. Soc. Am. B 20, 2037 (2003).

22. D. Moss, H. M. van Driel, and J. E. Sipe, Opt. Lett. 14, 57 (1989).

23. R. Claps, V. Raghunathan, D. Dimitropoulos, and B. Jalali, Opt. Express 12, 2774 (2004).

24. H. Tsang, C. Wong, T. Liang, I. Day, S. Roberts, A. Harpin, J. Drake, and M. Asghari, Appl. Phys. Lett. 80, 416 (2002).

25. Y. Ding, H. Ou, and C. Peucheret, Opt. Lett. 38, 2732 (2013). 


\section{FULL REFERENCES}

1. H. Rong, R. Jones, A. Liu, O. Cohen, D. Hak, A. Fang, and M. Paniccia, "A continuous-wave raman silicon laser," Nature 433, 725-728 (2005).

2. J. Leuthold, C. Koos, and W. Freude, "Nonlinear silicon photonics," Nat. Photon. 4, 535 (2010).

3. M. A. Foster, A. C. Turner, M. Lipson, and A. L. Gaeta, "Nonlinear optics in photonic nanowires," Opt. Express 16, 1300-1320 (2008).

4. G. A. Siviloglou, S. Suntsov, R. El-Ganainy, R. Iwanow, G. I. Stegeman, D. N. Christodoulides, R. Morandotti, D. Modotto, A. Locatelli, C. De Angelis, F. Pozzi, C. R. Stanley, and M. Sorel, "Enhanced thirdorder nonlinear effects in optical algaas nanowires," Opt. Express 14, 9377-9384 (2006).

5. R. Salem, M. A. Foster, A. C. Turner, D. F. Geraghty, M. Lipson, and A. L. Gaeta, "Signal regeneration using low-power four-wave mixing on silicon chip," Nat. Photon. 2, 35-38 (2008).

6. I.-W. Hsieh, X. Chen, X. Liu, J. I. Dadap, N. C. Panoiu, C.-Y. Chou, F. Xia, W. M. Green, Y. A. Vlasov, and R. M. Osgood, "Supercontinuum generation in silicon photonic wires," Opt. Express 15, 15242-15249 (2007).

7. K. Guo, E. N. Christensen, J. B. Christensen, J. G. Koefoed, D. Bacco, Y. Ding, H. Ou, and K. Rottwitt, "High coincidence-to-accidental ratio continuous-wave photon-pair generation in a grating-coupled silicon strip waveguide," Applied Physics Express 10, 062801 (2017).

8. M. A. Foster, A. C. Turner, J. E. Sharping, B. S. Schmidt, M. Lipson, and A. L. Gaeta, "Broad-band optical parametric gain on a silicon photonic chip," Nature 441, 960-963 (2006).

9. I. Agha, M. Davanço, B. Thurston, and K. Srinivasan, "Low-noise chipbased frequency conversion by four-wave-mixing bragg scattering in sin x waveguides," Opt. lett. 37, 2997-2999 (2012).

10. C. Koos, L. Jacome, C. Poulton, J. Leuthold, and W. Freude, "Nonlinear silicon-on-insulator waveguides for all-optical signal processing," Opt. Express 15, 5976 (2007).

11. Q. Lin, O. J. Painter, and G. P. Agrawal, "Nonlinear optical phenomena in silicon waveguides: modeling and applications," Opt. Express 15, 16604-16644 (2007).

12. S. Afshar and T. Monro, "A full vectorial model for pulse propagation in emerging waveguides with subwavelength structures part i: Kerr nonlinearity," Opt. Express 17, 2298-2318 (2009).

13. S. Afshar, T. Monro, and C. M. de Sterke, "Understanding the contribution of mode area and slow light to the effective kerr nonlinearity of waveguides," Opt. Express 21, 18558-18571 (2013).

14. T. J. Duffin, M. P. Nielsen, F. Diaz, S. Palomba, S. A. Maier, and R. F. Oulton, "Degenerate four-wave mixing in silicon hybrid plasmonic waveguides," Optics letters 41, 155-158 (2016).

15. K. Gehlot and A. Sharma, "Optimization of si slot waveguide using approximate semi-analytical method," Opt. Express 24, 4722-4729 (2016).

16. L. An, H. Liu, Q. Sun, N. Huang, and Z. Wang, "Wavelength conversion in highly nonlinear silicon-organic hybrid slot waveguides," Appl. Opt. 53, 4886-4893 (2014).

17. G. P. Agrawal, Nonlinear Fiber Optics (Elsevier, 2006), 4th ed.

18. S. Afshar, W. Q. Zhang, H. Ebendorff-Heidepriem, and T. Monro, "Small core optical waveguides are more nonlinear than expected: experimental confirmation," Opt. Lett. 34, 3577-3579 (2009).

19. M. Kolesik and J. Moloney, "Nonlinear optical pulse propagation simulation: From maxwell's to unidirectional equations," Phys. Rev. E 70, 036604 (2004).

20. A. W. Snyder and J. Love, Optical waveguide theory (Springer, 2012).

21. J. Lægsgaard, N. A. Mortensen, and A. Bjarklev, "Mode areas and field-energy distribution in honeycomb photonic bandgap fibers," J. Opt. Soc. Am. B 20, 2037-2045 (2003).

22. D. Moss, H. M. van Driel, and J. E. Sipe, "Dispersion in the anisotropy of optical third-harmonic generation in silicon," Opt. Lett. 14, 57-59 (1989).

23. R. Claps, V. Raghunathan, D. Dimitropoulos, and B. Jalali, "Influence of nonlinear absorption on raman amplification in silicon waveguides," Opt. Express 12, 2774-2780 (2004).

24. H. Tsang, C. Wong, T. Liang, I. Day, S. Roberts, A. Harpin, J. Drake, and M. Asghari, "Optical dispersion, two-photon absorption and selfphase modulation in silicon waveguides at $1.5 \mu \mathrm{m}$ wavelength," Appl. Phys. Lett. 80, 416-418 (2002).

25. Y. Ding, H. Ou, and C. Peucheret, "Ultrahigh-efficiency apodized grating coupler using fully etched photonic crystals," Opt. Lett. 38, 2732-2734 (2013). 